\title{
EFFECTS OF CHEMOTHERAPY TREATMENT ON ANXIETY AND FATIGUE OF WOMEN WITH BREAST CANCER
}

Naiany Pereira', Vitor Marques¹, Rafael Moraes ${ }^{1}$, Rafael Alves¹, Fabíola Mamede¹, Weder Silva1, Wanderson Santos ${ }^{1}$, Carlos Vieira ${ }^{1}$

${ }^{1}$ College of Physical Education and Dance, Universidade de Goiás - Goiânia (GO), Brazil.

Breast cancer is among the main types of cancer in the world. One of the treatment alternatives is chemotherapy, which in turn can cause antagonistic effects related to behavior, reflecting anxiety, and fatigue. Objective: The aim of this study was to evaluate the effects of chemotherapy treatment on anxiety and fatigue in women with breast cancer. Methods: A total of 37 women participated in the study and were distributed into two groups, namely, 19 undergoing chemotherapy for breast cancer (52 \pm 13.11 years) and 18 apparently healthy (55.8 \pm 8.37 years). All volunteers answered questions from the questionnaire related to the Piper Fatigue Scale, composed of 22 items subdivided into 4 distinct subjective dimensions, namely, affective, sensory, cognitive, and behavioral. Anxiety was assessed using the IDATE anxiety scale, an instrument that consists of two separate self-report scales: state of anxiety and trace state, each has 20 statements in which the subjects must describe how they feel. The significance level was set a priori at $\mathrm{p}<0.05$. Additionally, to examine the magnitude effect of chemotherapy treatment, Cohen's d effect size (ES) was calculated from the difference between group scores divided by the pooled standard deviation of 20 . The obtained $d$ values were used to define the chemotherapy treatment effect as trivial $(\mathrm{d}<0.2)$, small $(0.2 \leq \mathrm{d}<0.5)$, medium $(0.5 \leq \mathrm{d}<0.8)$, and large $(\mathrm{d} \geq 0.8)$. Results: There was no difference in the levels of fatigue and anxiety between the groups ( $p>0.05)$. Behavior fatigue ( $p=0.08, E S=0.56)$, affective fatigue ( $p=0.18$, $E S=0.44)$, sensory fatigue ( $p=0.09, E S=0.55)$, cognitive fatigue $(p=0.34, E S=0.31)$, general fatigue $(p=0.09, E S=0.56)$, anxiety state $(p=0.08, E S=0.56)$, and anxiety trait $(p=0.92, E S=0.03)$. Conclusion: The results of this study show that chemotherapy treatment between the third and fourth cycles does not directly affect anxiety and fatigue in women with breast cancer.

Keywords: Breast Cancer; Chemotherapy Treatment; Anxiety; Fatigue; Women. 